\title{
SPREAD SPECTRUM FOR INTERFEROMETRIC AND MAGNETIC RESONANCE IMAGING
}

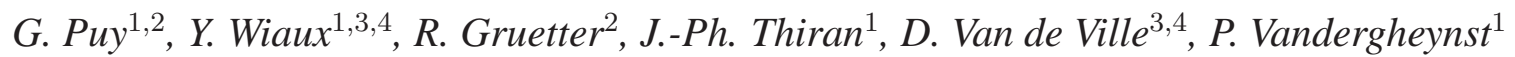 \\ ${ }^{1}$ Institute of Electrical Engineering, Ecole Polytechnique Fédérale de Lausanne (EPFL), CH-1015 Lausanne, Switzerland \\ ${ }^{2}$ Institute of the Physics of Biological Systems, Ecole Polytechnique Fédérale de Lausanne (EPFL), CH-1015 Lausanne, Switzerland \\ ${ }^{3}$ Institute of Bioengineering, Ecole Polytechnique Fédérale de Lausanne (EPFL), CH-1015 Lausanne, Switzerland \\ ${ }^{4}$ Department of Radiology and Medical Informatics, University of Geneva (UniGE), CH-1211 Geneva 14, Switzerland
}

\begin{abstract}
We consider images probed through incomplete and noisy Fourier coverages, both in the context of radio interferometry (RI) and of magnetic resonance imaging (MRI). We show that the quality of signal reconstruction can be significantly enhanced by the introduction of a linear chirp modulation, which induces a spread spectrum phenomenon.
\end{abstract}

Index Terms - image processing, compressed sensing, radio interferometry, magnetic resonance imaging

\section{COMPRESSED SENSING}

It is well-known that a large variety of natural signals are sparse or compressible in multi-scale dictionaries, such as wavelet frames. A band-limited signal may be expressed as the $N$-dimensional vector of its values sampled at the Nyquist-Shannon rate. By definition, a signal is sparse or compressible in some basis if its expansion contains only a small number $K \ll N$ of non-zero or significant coefficients, respectively. The theory of compressed sensing demonstrates that a small number $M \ll N$ of random measurements, in a sensing basis incoherent with the sparsity basis, will suffice for an accurate and stable reconstruction of such signals [1]. The basic framework proposes to solve the Basis Pursuit (BP) minimization problem for the signal recovery. This problem regularizes the originally ill-posed inverse problem by an explicit sparsity prior on the signal. In the presence of noise, the so-called Basis Pursuit denoise $\left(\mathrm{BP}_{\epsilon}\right)$ problem is the minimization of the $\ell_{1}$ norm of the coefficients of the signal in the sparsity basis $\Psi$ under a constraint on the $\ell_{2}$ norm of the residual noise.

In particular, random Fourier measurements of a signal sparse in real space represent a good sensing procedure. The mutual coherence $\mu(\mathrm{F}, \Psi)$ between the Fourier basis $\mathrm{F}$ and the sparsity basis $\psi$ may be defined as the maximum complex modulus of the scalar product between vectors of the two bases. In other words this mutual coherence identifies with the maximum complex modulus of the Fourier coefficient values of the sparsity basis vectors. It plays an essential role in the signal reconstruction quality as, for fixed $M$, the sparsity recovered increases with the mutual incoherence, i.e. the inverse of the coherence, as $K \propto \mu^{-2}(\mathrm{~F}, \Psi)$. The incoherence is maximum between the Fourier basis and the real space basis identified by a sparsity matrix $\psi \equiv \Delta$ made up of unit spikes: $\mu(\mathrm{F}, \Delta)=N^{-1 / 2}$.

\section{RADIO INTERFEROMETRY}

Aperture synthesis in RI is a powerful technique in astronomy, allowing observations of the sky with otherwise inaccessible angular resolutions and sensitivities [2]. In this context, the portion of the celestial sphere around the pointing direction tracked by a radio telescope array during observation defines the original real astrophysical signal or image probed $x$. The field of view (FOV) observed is limited by a primary beam $A^{\left(t_{0}\right)}$ with a support of size identified by $t_{0} \in \mathbb{R}_{+}$. Standard interferometers are characterized by a small FOV, so that the signal and the primary beam are assumed to be planar. Intensity signals respectively read as scalar functions $x(\boldsymbol{l})$ and $A^{\left(t_{0}\right)}(\boldsymbol{l})$ of the position $\boldsymbol{l} \in \mathbb{R}^{2}$, with components $(l, m)$. Each telescope pair at one instant of observation identifies a baseline defined as the relative position between the two telescopes. To each baseline $\boldsymbol{b}_{\lambda} \in \mathbb{R}^{3}$, with components $(u, v, w)$ in units of the signal emission wavelength $\lambda$, is associated one measurement called visibility. In the simplest setting one also considers baselines with negligible component $w$ in the pointing direction of the instrument. Under this additional assumption, if the signal is made up of incoherent sources, each visibility corresponds to the value of the Fourier transform of the signal multiplied by the primary beam at a spatial frequency $\boldsymbol{u} \in \mathbb{R}^{2}$, identified by the components $(u, v)$ of the baseline projection on the plane of the signal. Radio-interferometric data are thus identified by incomplete and noisy measurements in the Fourier plane. In the perspective of the reconstruction of the original image, these data define an ill-posed inverse problem.

In the present work, we raise the important problem of the dependence of the image reconstruction quality as a function of the sparsity basis, or more generally the sparsity dictionary (see also [3]). The larger the typical size, in real space, of 
the waveforms constituting the dictionary in which the signal is sparse or compressible, the smaller their extension in the Fourier plane, and the smaller the incoherence between the sparsity and sensing dictionaries. In the context of compressed sensing, a loss of incoherence leads to a degradation of the reconstruction quality for a given sparsity $K$ and a given number $M$ of random measurements.

The detailed structure of radio-interferometric measurements might actually provide a natural response to this issue. The approximation of baselines with negligible component $w$ is a key assumption in order to identify visibilities with Fourier measurements of the original signal. This approximation actually sets a strong constraint on the FOV probed by the interferometer, requiring that it is small enough, not only for the planar approximation of the signal but also in order to neglect the complete effect of the component $w$ in the visibilities. We relax this approximation and consider radio interferometers with small FOV and baselines with nonnegligible component $w$. Relying on the flexibility of realistic baseline distributions, we make the simplifying assumption that all baselines have the same component $w$. In this context, each visibility at spatial frequency $\boldsymbol{u}$ identifies with the Fourier transform of a complex signal obtained as the product of the original planar signal multiplied by the primary beam $A^{\left(t_{0}\right)} x(\boldsymbol{l})$ with a linear chirp $C^{(w)}(|\boldsymbol{l}|)=\mathrm{e}^{\mathrm{i} \pi w|\boldsymbol{l}|^{2}}$, where the norm $|\boldsymbol{l}|$ identifies the distance to the center of the image: $y(\boldsymbol{u})=C^{\left.(\widehat{w})^{\left(t_{0}\right.}\right)} x(\boldsymbol{u})$. In the Fourier plane, the modulation amounts to the convolution of the Fourier transform of the chirp with that of the signal multiplied by the primary beam $C^{(w) A^{\left(t_{0}\right)}} x=\widehat{C^{(w)}} \star \widehat{A^{\left(t_{0}\right)} x}$. This convolution inevitably spreads the two-dimensional sample power spectrum of the signal multiplied by the primary beam, i.e. the square modulus of its Fourier transform, while preserving its overall norm. This spread spectrum phenomenon increases the incoherence between the sparsity and sensing dictionaries.

\section{DICTIONARIES AND INVERSE PROBLEM}

The band-limited functions considered are completely identified by their Nyquist-Shannon sampling on a discrete uniform grid of $N=N^{1 / 2} \times N^{1 / 2}$ points $\boldsymbol{l}_{i} \in \mathbb{R}^{2}$ in real space with $1 \leq i \leq N$. The sampled signal is denoted by a vector $\boldsymbol{x} \in \mathbb{R}^{N} \equiv\left\{x_{i} \equiv x\left(\boldsymbol{l}_{i}\right)\right\}_{1 \leq i \leq N}$ and the primary beam is denoted by the vector $\boldsymbol{A}^{\left(t_{0}\right)} \in \mathbb{R}^{N} \equiv\left\{A_{i}^{\left(t_{0}\right)} \equiv\right.$ $\left.A^{\left(t_{0}\right)}\left(\boldsymbol{l}_{i}\right)\right\}_{1 \leq i \leq N}$. The chirp is complex and reads as the vector $\boldsymbol{C}^{(w)} \in \mathbb{C}^{N} \equiv\left\{C_{i}^{(w)} \equiv C^{(w)}\left(\left|\boldsymbol{l}_{i}\right|\right)\right\}_{1 \leq i \leq N}$. Because of the assumed finite FOV, the functions may equivalently be described by their complex Fourier coefficients on a discrete uniform grid of $N=N^{1 / 2} \times N^{1 / 2}$ spatial frequencies $\boldsymbol{u}_{i}$ with $1 \leq i \leq N$. This grid is limited at some maximum frequency defining the band limit.

We assume that the spatial frequencies $\boldsymbol{u}$ probed by all telescope pairs during the observation belong to the discrete grid of points $\boldsymbol{u}_{i}$. The Fourier coverage provided by the $M / 2$ spatial frequencies probed $\boldsymbol{u}_{b}$, with $1 \leq b \leq M / 2$, can simply be identified by a binary mask in the Fourier plane equal to 1 for each spatial frequency probed and 0 otherwise. The visibilities measured may be denoted by a vector of $M / 2$ complex Fourier coefficients $\boldsymbol{y} \in \mathbb{C}^{M / 2} \equiv\left\{y_{b} \equiv\right.$ $\left.y\left(\boldsymbol{u}_{b}\right)\right\}_{1 \leq b \leq M / 2}$, corresponding to $M$ real measures, and possibly affected by complex noise of astrophysical or instrumental origin, identified by the vector $\boldsymbol{n} \in \mathbb{C}^{M / 2} \equiv\left\{n_{b} \equiv\right.$ $\left.n\left(\boldsymbol{u}_{b}\right)\right\}_{1 \leq b \leq M / 2}$. Relying on the flexibility of realistic baseline distributions, we also assume that the spatial frequencies $\boldsymbol{u}_{b}$ probed arise from a uniform random selection of Fourier frequencies. As for the assumption of constant $w$, this allows us to discard considerations related to specific interferometers. It also allows us to place our discussion in a setting which complies directly with the requirement of the theory of compressed sensing for random measurements.

In this discrete setting, the Fourier coverage is in general incomplete in the sense that the number of real constraints $M$ is smaller than the number of unknowns $N: M<N$. An illposed inverse problem is thus defined for the reconstruction of the signal $\boldsymbol{x}$ from the measured visibilities $\boldsymbol{y}$ :

$$
\boldsymbol{y} \equiv \Phi^{\left(w, t_{0}\right)} \boldsymbol{x}+\boldsymbol{n} \text { with } \Phi^{\left(w, t_{0}\right)} \equiv \operatorname{MFC}^{(w)} \mathrm{A}^{\left(t_{0}\right)},
$$

where the matrix $\Phi^{\left(w, t_{0}\right)} \in \mathbb{C}^{(M / 2) \times N}$ identifies the complete linear relation between the signal and the visibilities. The matrix $\mathrm{A}^{\left(t_{0}\right)} \in \mathbb{R}^{N \times N} \equiv\left\{A_{i j}^{\left(t_{0}\right)} \equiv A_{i}^{\left(t_{0}\right)} \delta_{i j}\right\}_{1 \leq i, j \leq N}$ is the diagonal matrix implementing the primary beam. The matrix $C^{(w)} \in \mathbb{C}^{N \times N} \equiv\left\{C_{i j}^{(w)} \equiv C_{i}^{(w)} \delta_{i j}\right\}_{1 \leq i, j \leq N}$ is the diagonal matrix implementing the chirp modulation. The unitary matrix $\mathrm{F} \in \mathbb{C}^{N \times N} \equiv\left\{F_{i j} \equiv \mathrm{e}^{-2 \mathrm{i} \pi \boldsymbol{u}_{i} \cdot \boldsymbol{x}_{j}} / N^{1 / 2}\right\}_{1 \leq i, j \leq N}$ implements the discrete Fourier transform. The matrix $M \in$ $\mathbb{R}^{(M / 2) \times N} \equiv\left\{M_{b j}\right\}_{1 \leq b \leq M / 2 ; 1 \leq j \leq N}$ is the rectangular binary matrix implementing the mask characterizing the interferometer. It contains only one non-zero value on each line, at the index of the Fourier coefficient corresponding to each of the spatial frequencies probed $\boldsymbol{u}$.

For the sake of simplicity and without loss of generality, we consider simple astrophysical signals sparse in a dictionary of Gaussian waveforms, all with equal and fixed size given by a standard deviation $t \in \mathbb{R}_{+}$. The sparsity dictionary identifying Gaussian waveforms of size $t$ may be denoted by $\Psi \equiv \Psi^{(t)} \equiv \Gamma^{(t)}$. The sensing dictionary as seen from the sparsity dictionary itself therefore reads as $\Theta^{\left(w, t_{0}, t\right)} \equiv$ $\Phi^{\left(w, t_{0}\right)} \Gamma^{(t)} \equiv \operatorname{MFC}^{(w)} \mathbf{A}^{\left(t_{0}\right)} \Gamma^{(t)}$.

\section{SPREAD SPECTRUM AND COHERENCE}

We assume a Gaussian primary beam $A^{\left(t_{0}\right)}$ with a size identified by a standard deviation $t_{0}$. After normalization of the vectors of the sparsity and sensing dictionaries in $\ell_{2}$ norm, a simple analytical computation gives the mutual coherence 
between the sensing and sparsity dictionaries as:

$$
\mu\left(\mathrm{FC}^{(w)} \mathrm{A}^{\left(t_{0}\right)}, \Gamma^{(t)}\right)=\frac{2 t t_{0}}{t^{2}+t_{0}^{2}}\left[1+\left(\frac{2 \pi w t^{2} t_{0}^{2}}{t^{2}+t_{0}^{2}}\right)^{2}\right]^{-\frac{1}{2}}
$$

Analyzing the evolution of the coherence as a function of the parameters $t, t_{0}$, and $w$ in the continuous limit $N \rightarrow \infty$ is very enlightening. Firstly, when the size $t$ of the Gaussian waveforms tends to zero, the mutual coherence tends to zero. In the absence of chirp modulation and primary beam, this null value was expected as the modified sparsity dictionary identifies with the Dirac basis. Secondly, in the limit of an infinite chirp rate, the coherence also tends to its minimum null value independently of the size $t$ of the Gaussian waveforms. This limit provides a strong result in the sense that the incoherence lost by considering a sparsity dictionary of Gaussian waveforms of arbitrary non-zero size $t$ may be completely recovered thanks to a chirp modulation with high enough chirp rate $w$. This result suggests the universality of the spread spectrum phenomenon according to which the quality of the $\mathrm{BP}_{\epsilon}$ reconstruction can be rendered independent of the sparsity dictionary for a large enough component $w$ of the baselines.

\section{SIMULATIONS AND RESULTS}

The astrophysical signals $x$ considered are built as the superposition of $K=10$ Gaussian waveforms, with random positions, and random central value in the interval $[0,1]$ in some arbitrary intensity units. The signals are sampled as images $\boldsymbol{x}$ on a grid of $N=64 \times 64=4096$ pixels. Fields of view $L=L^{1 / 2} \times L^{1 / 2}$ with $L^{1 / 2}$ not larger than several degrees of angular opening may be considered for the approximation of a planar signal to be valid. A primary beam $A^{\left(t_{0}\right)}$ with a full width at half maximum (FWHM) $2 t_{0}(2 \ln 2)^{1 / 2}=L^{1 / 2}$ is also considered and with unit value at the center. In the discrete setting the inverse problem is transparent to the precise value of the FOV so that we do not need to fix it. Observations are considered for $M / 2$ complex visibilities with $M / 2 \in\{50,100,200,300,400,500,1000\}$ corresponding to coverages between 1 and 25 per cent of the Fourier plane. A small amount of instrumental noise is also added as independent identically distributed Gaussian noise.

The size $t$ of the Gaussian waveforms may be written in terms of a discrete size $t_{d}$ as $t=t_{d} L^{1 / 2} / \pi N^{1 / 2}$. We consider the values $t_{d}=2$ and $t_{d}=16$. For $t_{d}=2$ the approximate band limit of the signal is around the same value as that accessible on the grids considered, while for $t_{d}=16$ it is much smaller. Original signal samples are reported in Fig. 1. The component $w$ may also be written in terms of a discrete component $w_{d}$ as $w=w_{d} N^{1 / 2} / L$. We consider the values $w_{d}=0$ and $w_{d}=1$. The case $w_{d}=0$ identifies baselines with negligible component $w$. The case $w_{d}=1$ identifies baselines with non-negligible and constant component $w$, corresponding to a linear chirp modulation with a band limit equal to that accessible on the grids. In other words the component $w$ is a factor $2 / L^{1 / 2}$ larger than the maximum value of the components $u$ and $v$ in the plane of the signal, so that for the small FOV assumed the baselines are strongly aligned with the pointing direction. Let us emphasize that a specific design of interferometer arrays should be studied in order to obtain such an alignment, or larger FOV should be considered.

A number of 30 simulations are generated for each value of $t_{d}, w_{d}$ and $M$ considered. The visibilities are simulated and the signals are reconstructed through the $\mathrm{BP}_{\epsilon}$ problem, which is solved by convex optimization. The quality of reconstruction is analyzed in terms of the signal-to-noise ratio SNR of the reconstructions multiplied by the primary beam. We actually compare two different settings for the reconstructions. In the first setting, called $\Gamma \mathrm{BP}_{\epsilon}$, we assume that the Gaussian waveform dictionary with appropriate $t$ is known, and we use it explicitly as sparsity dictionary: $\Psi \equiv \Gamma^{(t)}$. As a consequence, the $\Gamma \mathrm{BP}_{\epsilon}$ problem deals with the best possible sparsity value $K=10$. The reconstructions in the absence $\left(w_{d}=0\right)$ and in the presence $\left(w_{d}=1\right)$ of the chirp modulation are respectively denoted by $\Gamma \mathrm{BP}_{\epsilon} 0$ and $\Gamma \mathrm{BP}_{\epsilon} 1$. It is the precise setting in which we just brought up the spread spectrum phenomenon. In the second setting, called $\triangle \mathrm{BP}_{\epsilon}$, we assume that the sparsity dictionary is the real space basis: $\Psi \equiv \Delta$. As a consequence, the $\Delta \mathrm{BP}_{\epsilon}$ problem deals with the best possible coherence value. However, the sparsity computed in real space increases drastically with the size of the Gaussian waveforms, suggesting that the reconstruction quality should clearly decrease when the Gaussian size increases. The reconstructions in the absence $\left(w_{d}=0\right)$ and in the presence $\left(w_{d}=1\right)$ of the chirp modulation are respectively denoted by $\triangle \mathrm{BP}_{\epsilon} 0$ and $\triangle \mathrm{BP}_{\epsilon} 1$. But the mutual coherence here remains unaffected by the chirp modulation, so that this modulation should fail to enhance the reconstruction quality in this case. It is known that this $\triangle \mathrm{BP}_{\epsilon}$ approach provides reconstruction qualities very similar to the standard CLEAN algorithm currently used in RI.

The results of the analysis are reported in Fig. 1. The SNR's of the $\triangle \mathrm{BP}_{\epsilon} 0$ reconstructions are significantly smaller than those of the $\Gamma \mathrm{BP}_{\epsilon} 0$ reconstructions. Moreover as expected, the $\triangle \mathrm{BP}_{\epsilon} 1$ approach does not profit from the spread spectrum phenomenon relative to the $\triangle \mathrm{BP}_{\epsilon} 0$ approach. The SNR's of the $\Gamma \mathrm{BP}_{\epsilon} 1$ reconstructions are significantly larger than those of the $\Gamma \mathrm{BP}_{\epsilon} 0$ reconstructions. This is the spread spectrum phenomenon related to the reduction of the mutual coherence in the presence of the chirp modulation. Moreover, the SNR's of the $\Gamma_{\mathrm{BP}} 1$ reconstructions are essentially independent of the sparsity dictionary identified by $t_{d}$. This supports very strongly the principle of universality of the spread spectrum phenomenon relative to the sparsity dictionary, in perfect agreement with our theoretical considerations. 

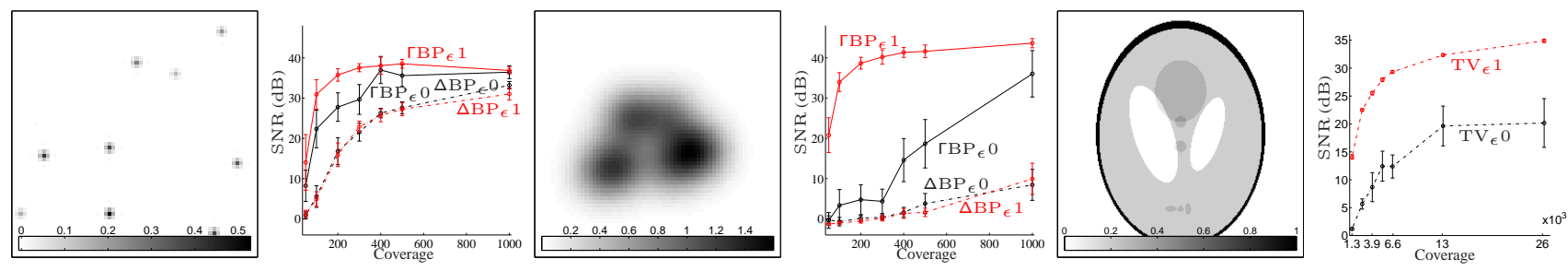

Fig. 1. Interferometry: the first and and third panels from the left represent original astrophysical signal samples with sizes $t_{d}=2$ and $t_{d}=16$ of the Gaussian waveforms respectively. The second and fourth panels from the left represent the SNR of reconstruction as a function of the coverage for $t_{d}=2$ and $t_{d}=16$ respectively. Magnetic resonance imaging: the second panel from the right represents original Shepp-Logan phantom image. The first panel from the right represents the SNR of reconstruction as a function of the coverage. All SNR curves in the three corresponding panels represent the mean SNR over 30 simulations, and the vertical lines identify the error at 1 standard deviation.

\section{MAGNETIC RESONANCE IMAGING}

In the standard setting, data acquired in MRI also provide an incomplete and noisy coverage of the Fourier plane, also called $\boldsymbol{k}$-space, of the original two-dimensional image $x$ of interest. However the use of quadratic magnetic fields was advocated for various purposes, notably for reducing aliasing artifacts in the signal reconstruction [4]. This technique is known under the name of phase scrambling and exactly corresponds to alter the original image by the linear chirp modulation that we considered in RI. It therefore obviously appears that the quality of sparse signal reconstruction can equivalently be enhanced in this context through the spread spectrum phenomenon.

As an illustration, we consider the Shepp-Logan phantom in some arbitrary intensity units as original image $x$ sampled on a grid of $N=256 \times 256=65536$ pixels on some FOV $L=L^{1 / 2} \times L^{1 / 2}$. An ill-posed inverse problem of the form (1) is posed. In this case, no explicit sparsity basis is considered, i.e. $\psi \equiv \Delta$, and no primary beam applies, i.e. $\left.\mathrm{A}^{\left(t_{0}\right)} \in \mathbb{R}^{N \times N} \equiv \delta_{i j}\right\}_{1 \leq i, j \leq N}$. Acquisitions are considered for a generic random distribution of $M / 2$ complex Fourier coefficients with $M / 2 \in$ $\{1311,2621,3932,5243,6554,13107,26214\}$ corresponding to coverages between 2 and 40 per cent of the Fourier plane. A small amount of instrumental noise is again added as independent identically distributed Gaussian noise.

By definition, the image exhibits exactly sparse magnitude of the gradient. An explicit theoretical computation of the incoherence was not achieved in this case. But the incoherence between the Fourier sensing basis and the space of the magnitude of the gradient of the signal, which may be thought of as a sparsity basis made up of step functions, is clearly not optimal. The introduction of a linear chirp modulation will therefore automatically spread the spectrum of the image and increase the incoherence between the sensing and sparsity bases. We consider the values $w_{d}=0$ and $w_{d}=1$ for the linear chirp. The image is reconstructed by solving the well- known analysis-based Total Variation $\left(\mathrm{TV}_{\epsilon}\right)$ problem, which consists in the minimization of the $\ell_{1}$ norm of the magnitude of the gradient of the signal under a constraint on the $\ell_{2}$ norm of the residual noise. This problem is again solved by convex optimization. The reconstructions in the absence $\left(w_{d}=0\right)$ and in the presence $\left(w_{d}=1\right)$ of the chirp modulation are respectively denoted by $\mathrm{TV}_{\epsilon} 0$ and $\mathrm{TV}_{\epsilon} 1$. Again, a number of 30 simulations are generated for each value of $w_{d}$ and $M$ considered. The quality of reconstruction is analyzed in terms of the signal-to-noise ratio SNR of the reconstructions. The results of the analysis are reported in Fig. 1. These results confirm the efficiency of the spread spectrum phenomenon in increasing the quality of signal reconstruction in MRI.

\section{CONCLUSION}

We have shown, on the basis of the theory of compressed sensing and through simulations, that a linear chirp modulation can drastically enhance the reconstruction quality of signals in RI and MRI, thanks to a related spectrum phenomenon. While this modulation can be simply obtained through quadratic magnetic fields in MRI, it will require the study of specific designs of arrays in RI.

\section{REFERENCES}

[1] Candès E. J., 2006, in Sanz-Solé M., Soria J., Varona J. L., Verdera J., eds, Proc. Int. Congress Math. Vol. 3. Euro. Math. Soc. Zürich, p. 1433

[2] Thompson A. R., Moran J. M., Swenson G. W. Jr, 2004, Interferometry and Synthesis in Radio Astronomy. WILEY-VCH Verlag GmbH \& Co. KGaA, Weinheim

[3] Wiaux Y., Puy G., Y. Boursier, Vandergheynst P., 2009, MNRAS, 400, 1029

[4] Ito S., Yamada Y., 2008, Magn. Reson. Med., 60, 422 